\title{
Supramolecular columns of hexabenzocoronenes on copper and gold (111) surfaces
}

\author{
P. Ruffieux, ${ }^{1, *}$ O. Gröning, ${ }^{1,2}$ M. Bielmann, ${ }^{1}$ C. Simpson, ${ }^{3}$ K. Müllen, ${ }^{3}$ L. Sclllapbach,,${ }^{1,2}$ and P. Gröning ${ }^{1}$ \\ ${ }^{1}$ Physics Department, University of Fribourg, Pérolles, CH-1700 Fribourg, Switzerland \\ ${ }^{2}$ Swiss Federal Laboratories for Materials Testing and Research, Überlandstrasse 129, 8600 Dübendorf, Switzerland \\ ${ }^{3}$ Max-Planck-Institut fïr Polymerforschung, Ackermannweg 10, 55128 Mainz, Germany
}

(Received 28 May 2002; published 5 August 2002)

\begin{abstract}
We report on the growth of supramolecular columns of polyaromatic hydrocarbons on $\mathrm{Au}(111)$ and $\mathrm{Cu}(111)$ single-crystal surfaces. The lateral separation of the columns was found to depend on the substrate and is determined by the commensurately formed superlattice of the first molecular monolayer. X-ray photoelectron diffraction in combination with low-energy electron diffiaction reveals stack growth with small lateral offsets from the column axis but with conservation of the molecular orientation. The mechanism of column growth is explained by simulation results of the intermolecular interaction assuming a Lennard-Jones potential. The size of hexabenzocoronene and its ability to condense into one-dimensional supramolecular structures make it an ideal candidate for the accommodation and the positioning of functional groups to form a functional molecular assembly.
\end{abstract}

DOI: $10.1103 /$ PhysRevB.66.073409

PACS number(s): 81.16.Fg, 81.07.Nb

For the realization of molecule-based electronic devices the understanding and control of intermolecular and molecule-substrate interactions are crucial. ${ }^{1-4}$ Directed supramolecular aggregation is achieved by adjusting intermolecular interactions and choosing appropriate substrates., ${ }^{3,4}$ Hexa-peri-hexabenzocoronene $\left[\mathrm{C}_{42} \mathrm{H}_{18}(\mathrm{HBC})\right]$ is a polycyclic aromatic hydrocarbon and can be viewed as being discshaped, hydrogen terminated two-dimensional graphite sections. The molecules are large enough to accommodate functional groups and therefore, the study of their growth mode and the control of it are of great importance. For the hexaalkyl-substituted derivatives of HBC high solubility and the ability to self-organize into a columnar mesophase in organic solvents has been shown. ${ }^{5,6}$ These structures are onedimensional conductors with a very high charge carrier mobility. ${ }^{6-8}$ However, the deposition of similar onedimensional structures at surfaces would be desirable in view of a controlled positioning and orientation of such columns.

In this work we report on the growth mode of thin films of the insoluble HBC prepared by vacuum sublimation onto $\mathrm{Au}(111)$ and $\mathrm{Cu}(111)$. We show that the two-dimensional structural information of the first molecular monolayer is transmitted to molecules of the following layers yielding columnar stacks of $\mathrm{HBC}$ with substrate-dependent stack separation. The observed aggregation into columnar structures is completely different from the well-known herringbone bulk structure. The combination of low-energy electron diffraction (LEED) and x-ray photoelectron diffraction (XPD) allows the determination of the intermolecular ordering and the orientation of the adsorbed molecules within the columns. XPD in combination with single-scattering cluster (SSC) calculation has been proven adequate for near-surface and adsorbates structure determination of carbon allotropes $^{10,11}$ and is used here for the determination of the intracolumn molecular ordering.

Experiments were performed in an OMICRON photoelectron spectrometer modified for motorized sequential anglescanning data acquisition having a base pressure in the low $10^{-11}$ mbar range. X-ray photoelectron spectra and diffrac- tion patterns were measured using $\mathrm{Mg} \cdot \mathrm{K} \alpha$ ( $h \nu$ $=1253.6 \mathrm{eV}$ ) radiation. The preparation of $\mathrm{Au}(111)$ and $\mathrm{Cu}(111)$ single crystals was done by repeated cycles of $\mathrm{Ar}^{+}$ ion sputtering and subsequent annealing to $600^{\circ} \mathrm{C}$ and $550^{\circ} \mathrm{C}$, respectively. $\mathrm{HBC}$ was evaporated from a resistively heated stainless steel crucible. After outgassing below the sublimation temperature, molecules were evaporated at $400^{\circ} \mathrm{C}$ with the chamber pressure staying below 1 $\times 10^{-9}$ mbar.

A monolayer of HBC was prepared by evaporation of several layers on the clean surfaces and subsequent annealing above the sublimation temperature of $\mathrm{HBC}$, which results in a well ordered HBC monolayer, as confirmed by LEED. Figure 1 shows diffraction patterns of the simultaneously recorded $\mathrm{C} 1 \mathrm{~s}$ and substrate signals for a monolayer of $\mathrm{HBC}$ on $\mathrm{Au}(111)$ and $\mathrm{Cu}(111)$, respectively. Prominent maxima in the diffraction patterns can be interpreted as nearest-neighbor directions. ${ }^{12}$ For the $\mathrm{C} 1 \mathrm{~s}$ diffraction pattern the strongest maxima, originating from the $\mathrm{C}-\mathrm{C}$ bond directions, are found at angles close to $90^{\circ}$ off normal, indicating that molecules are adsorbed flat on the surface. The strongest maxima show the directions of the $\mathrm{C}-\mathrm{C}$ bonds; focusing in the direction of the next-nearest neighbors yields weaker features rotated by $30^{\circ}$ to the principal maxima. Comparison to the substrate diffraction pattern then allows the determination of the molecular orientation with respect to the substrate lattice.

On $\mathrm{Au}(111)$ the molecular HBC monolayer film forms a hexagonal $\left(\begin{array}{cc}3 & 3 \\ -3 & 6\end{array}\right)$ structure, whereas that on $\mathrm{Cu}(111)$ shows a LEED pattern corresponding to a hexagonal arrangement of the molecules with the two possible domains $\left(\begin{array}{cc}5 & 1 \\ -1 & 6\end{array}\right)$ and $\left(\begin{array}{cc}6 & -1 \\ 1 & 5\end{array}\right)$. The combination of the results from XPD and LEED allows a complete picture of the molecular ordering in terms of intermolecular distance and molecule orientation (Fig. 2).

On $\mathrm{Au}(111) \mathrm{HBC}$ has its molecular axis (labeled $m$ in Fig. 2) parallel to the molecular lattice vector [01] with a nearestneighbor distance of $15.0 \AA$. On $\mathrm{Cu}(111)$ the molecules adsorb with the molecular axis rotated by, $9^{\circ}$ relative to the 


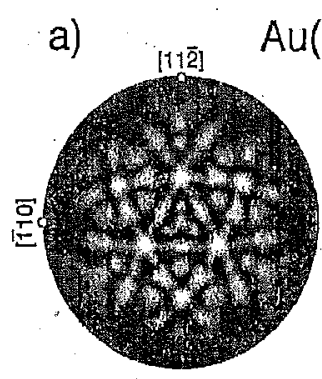

Au4f

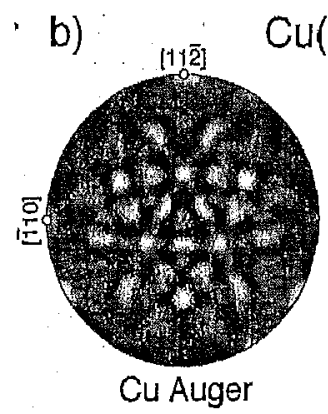

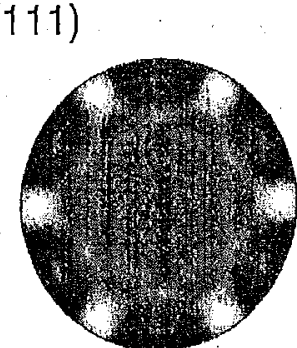

C1s

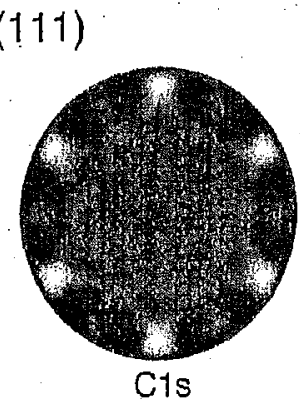

FIG. I. Experimental photoelectron diffraction patterns of substrate and monolayer film signal. Intensity plots are shown in stereographic projection with the center showing normal emission and the circle showing emission at $\vartheta=90^{\circ}$. White corresponds to highest intensity. (a) Monolayer of HBC on $\mathrm{Au}(11 \mathrm{l})$. Substrate Au4 $\mathrm{f}_{7 / 2}$ $\left(E_{\mathrm{kin}}=1170 \mathrm{eV}\right)$ and $\mathrm{Cls}\left(E_{\mathrm{kin}}=970 \mathrm{eV}\right)$ signal. (b) Monolayer film on $\mathrm{Cu}(111)$, Cu L $\mathrm{L}_{3} \mathrm{VV}\left(E_{\mathrm{kin}}=919 \mathrm{eV}\right)$, Cls $\left(E_{\mathrm{kin}}=970 \mathrm{eV}\right)$.

molecular lattice vectors and with a nearest-neighbor distance of $14.2 \AA$. Structural data of the monolayer systems are summarized in Table $\mathrm{I}$.

In general, the two-dimensional ordering depends on the interplay of the moleccule-substrate and the intermolecular interactions. The growth of commensurate superstructures requires a sufficiently stiong molecule-substrate interaction. This molecule-substrate interaction modulates the intermolecular potential with a contribution having the periodicity of the substrate lattice. If this contribution is strong enough, the equilibrium distance between two molecules in the first monolayer can be changed to a distance that is commensurate to the substrate lattice: Furthermore, molecules can be rotated away from the equilibrium orientation in the absence of the substrate interaction, for which in the case of HBC the molecular axis $m$ is aligned with the [01] direction. The equilibrium distance $d_{0}$ found from the intermolecular potential calculation for a molecular monolayer film is $14.1 \AA$ in the absence of substrate interaction (details of the calculation are given below), which is lower than the observed nearestneighbor distances of 15.0 and $14.2 \AA$ for the $\mathrm{Au}(111)$ and

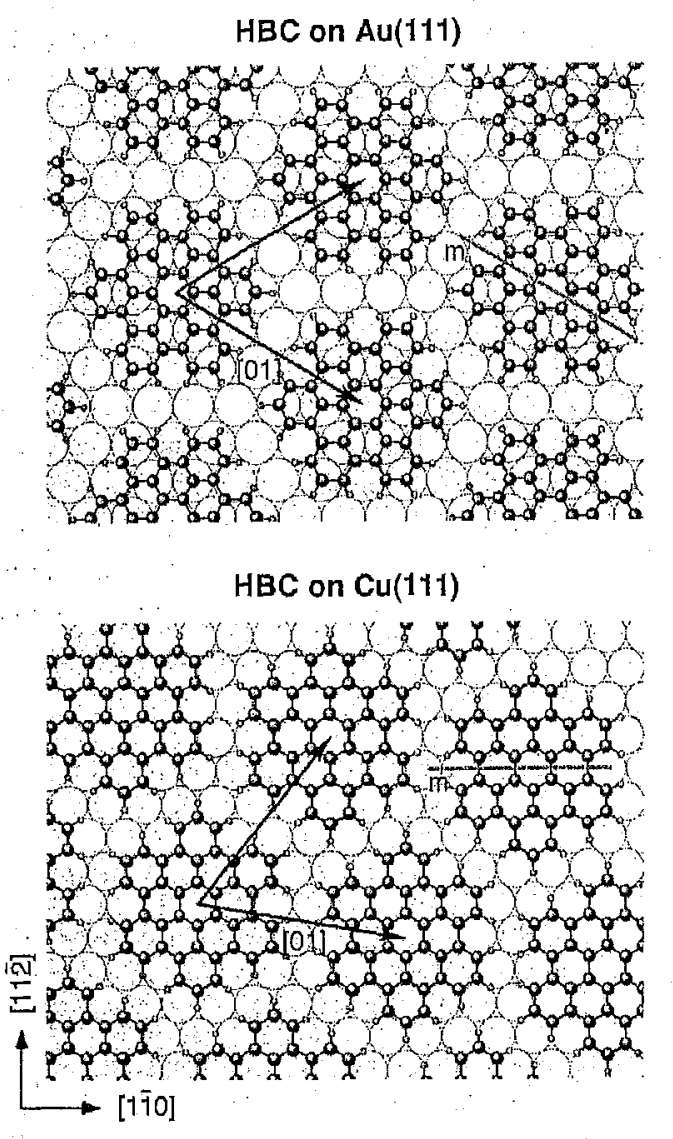

FIG. 2. Two dimensional ordering of HBC molecules on $\mathrm{Cu}(111)$ (a) and $\mathrm{Au}(111)$ (b). Lattice vectors of the substrate and the molecular film are indicated with arrows. Lines labeled $m$ indicate the molcular axis of HBC.

$\mathrm{Cu}(111)$ surfaces, respectively. The expansion of the molecular lattice by $\sim 6 \%$ on $\mathrm{Au}(111)$ and the molecule rotation of $9^{\circ}$ on $\mathrm{Cu}(111)$ indicate a rather strong molecule-substrate interaction on both investigated substrates resulting in films with different densities and molecule orientations.

In order to study the three-dimensional stacking of $\mathrm{HBC}$, subsequent layers have been evaporated onto the ordered monolayer systems. As evidenced by LEED, the superstructure remains the same as for the monolayer film. ${ }^{13}$ The XPD patterns of $\sim 10 \AA$ thick multilayers deposited on $\mathrm{Cu}(111)$ and $\mathrm{Au}(111)$ are displayed in Figs. 3(a) and 3(b). In contrast to the patterns of monolayer films, new features are expected to appear at nongrazing emission due to the appearance of new scatterers in the next higher layer. However, SSC calculations for the case where all second layer molecules have the same offset with respect to first layer molecules show much more distinct features near normal emission than the

TABLE I. Structural data of the two-dimensional $\mathrm{HBC}$ films on $\mathrm{Au}(111)$ and $\mathrm{Cu}(111)$. The \pm stands for the two possible domains on $\mathrm{Cu}(111)$.

\begin{tabular}{|c|c|c|c|c|}
\hline Substrate & Superstructure & $\begin{array}{c}\text { HBC }[01] \mathrm{rel} . \\
\text { to substrate }[11 \overline{2}]\end{array}$ & $\begin{array}{l}\text { Nearest-neighbor } \\
\text { distance }\end{array}$ & $\begin{array}{l}\text { Orientation of mol. } \\
\text { axis rel. to HBC [01] }\end{array}$ \\
\hline $\mathrm{Au}(111)$ & $(\sqrt{27} \times \sqrt{27}) R 30^{\circ}$ & $\therefore 60^{\circ}$ & $15.0 \AA$ & $0^{\circ}$ \\
\hline $\mathrm{Cu}(111)$ & $(\sqrt{31} \times \sqrt{31}) R \pm 8.95^{\circ}$ & $30 \pm 9^{\circ}$ & $14.2 \AA$ & $\pm 9^{\circ}$ \\
\hline
\end{tabular}




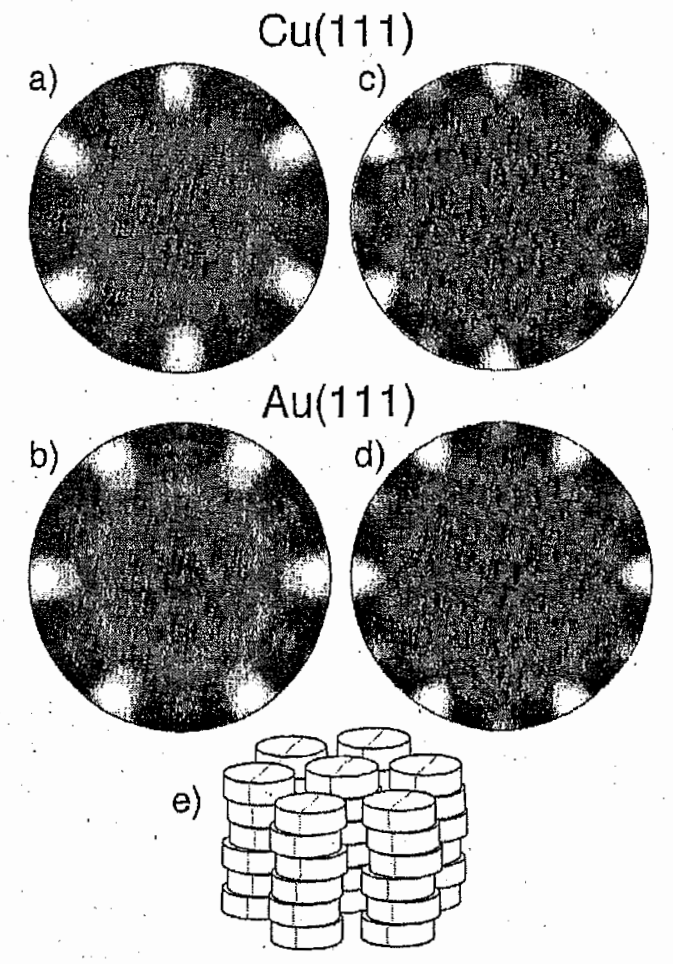

FIG. 3. Multilayer films $(n \simeq 3)$ of HBC. (a) Experimental XPD pattern of the C1s signal $\left(E_{\mathrm{kin}}=970 \mathrm{eV}\right.$ ) on $\mathrm{Cu}(111)$. (b) Exp. XPD pattern of $\mathrm{C} 1 \mathrm{~s}$ on $\mathrm{Au}(111)$. (c) SSC pattern calculated for various positions allowed at room temperature (see text). (d) Averaged SSC pattern for various shifts of subsequent molecules in the direction of the $\mathrm{C}-\mathrm{C}$ bond. Offsets for the individual calculations were in the range of 0.6 to $1.2 \AA$. (e) Proposed column structure.

experimental patterns. This low number and low anisotropy of features near normal emission of the experimental XPD pattern suggests a multitude of possible lateral offsets, a model which will be discussed in the last part. The intensity maxima at grazing emission remain unchanged, indicating the preservation of molecular orientation during film growth. The degree to which the orientation is preserved can be estimated from the variation of the anisotropy of the intensity in the XPD pattern. As at grazing emission surface sensitivity is highest, changes in anisotropy directly indicate the degree of orientational ordering of the topmost molecules of the stacks. The anisotropy of the intensity at grazing emission remains on the level of the monolayer system (0.18) for thicknesses of up to about $20 \AA$. For even higher stacks $(\sim 25 \AA)$ the anisotropy drops only to 0.14 . This is most likely due to an increasing misorder in the azimuthal orientation and not due to a tilting of the molecules, since it has been shown for multilayer films deposited on flat substrates, that the molecules lie flat for film thicknesses of up to $\sim 100 \AA .{ }^{14}$ The results from LEED and XPD suggest the growth of columns of flat lying molecules where the molecule orientation in the column is preserved.

The intermolecular potential has been calculated for different stacking positions of a $\mathrm{HBC}$ molecule on a completed monolayer using a Lennard-Jones potential of the type of $V_{i j}\left(r_{i j}\right)=4 \varepsilon_{i j}\left[\left(\sigma_{i j} / r_{i j}\right)^{12}-\left(\sigma_{i j} / r_{i j}\right)^{6}\right]$, which describes well nonbonded intermolecular interactions between hydrocarbons and fullerenes. ${ }^{15.16}$ The parameters $\varepsilon_{i j}$ and $\sigma_{i j}$ for

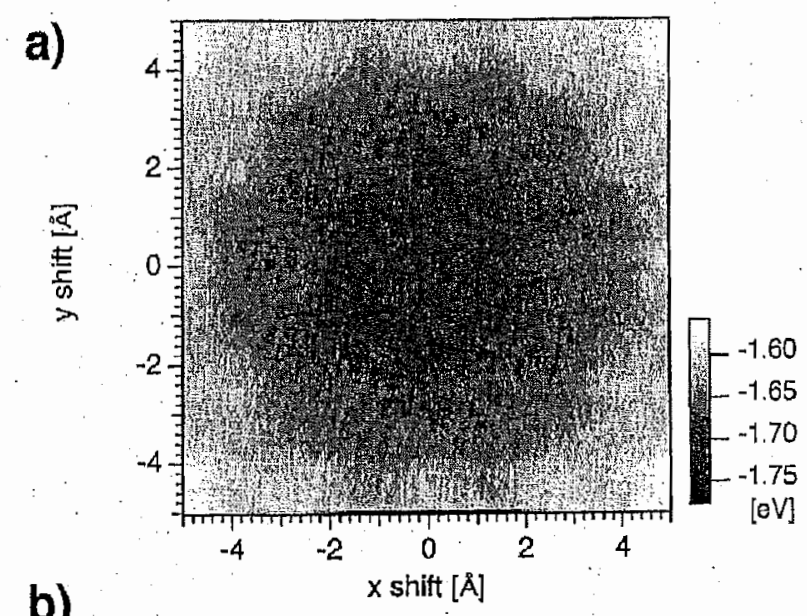

b)
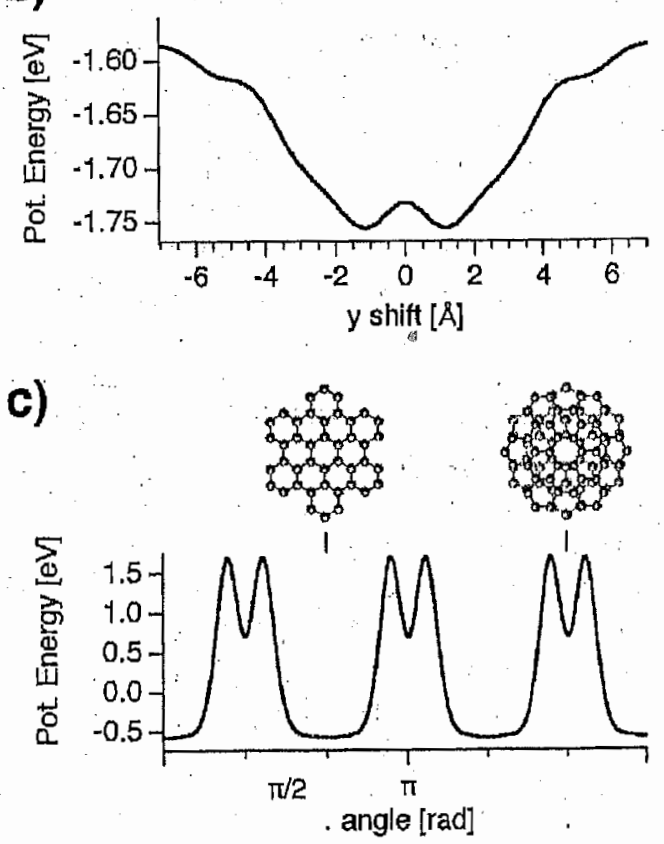

FIG. 4. Calculation of the intermolecular potential of a single $\mathrm{HBC}$ molecule on a completed monolayer $\mathrm{HBC} / \mathrm{Au}(111)$ film. The intermolecular equilibrium distance in $\mathrm{z}$ is found to be $3.4 \AA$. (a) Potential as a function of $x y$ shift with respect to the center of a molecule of the first layer. (b) Potential as function of $y$ shift at $x$ $=0$. (c) Interaction with nearest-neighbour molecules in the same layer as a function of molecule orientation.

$\mathrm{C}-\mathrm{C}$ and $\mathrm{H}-\mathrm{H}$. interactions are taken from the work of Stuart. ${ }^{15}$ The mixed parameters $\varepsilon_{\mathrm{CH}}$ and $\sigma_{\mathrm{CH}}$ are determined according to the Lorentz-Berthelot combining rules. ${ }^{17}$ Atom coordinates of the molecule were taken from the $\mathrm{x}$-ray analysis of Goddard et al. ${ }^{9}$ In order to determine the position a molecule would take when adsorbing on a completed monolayer, a HBC molecule and its six nearest-neighbors were taken at fixed positions as determined by LEED and XPD and one molecule of the second layer was used as probe. The position-dependent potential was calculated by summing up the interaction potentials of each of the atoms in the molecule of the second layer with all the atoms of the molecules of the first layer. A potential plot obtained in this way is shown in Fig. 4(a) showing the dependence on the $x y$ shift of the top molecule with respect to the molecule underneath. Coordinates for molecules of the first layer are taken for the 
$\mathrm{HBC} / \mathrm{Au}(111)$ superstructure (see Table I). The vertical equilibrium distance between two layers is $3.4 \AA$. The offset dependence of the intermolecular potential is dominated by the attractive van der Waals interaction which favors on axis columnar aggregation in order to maximize the overlapping of two molecules. On the other hand, the energetically favored sequence of stacked graphene sheets is $A B A$, where subsequent layers are shifted by $1.42 \AA$ relative to each other: This contribution can be seen as a $\sqrt{3} \times 1.42 \AA$ modulation of the potential and is responsable for the off-center position $(1.2 \AA)$ of the potential minimum. However, for a single molecule adsorbed in the second layer, the absolute potential minimum is found for an azimuthal rotation of $30^{\circ}$ relative to first-layer molecules (not shown). This orientation of the molecules becomes unfavorable if nearest-neighbors in the second layer are taken into account [Fig. 4(c)]. The facing hexagon corners of neighboring molecules are in the repulsive regime of the van der Waals interaction if molecules are not aligned to molecules of the first layer. This promotes the realignement during film growth and explains the observed conservation of the molecular orientation on multilayer samples. We performed SSC calculations for molecule stacking sequences according to the calculated intermolecular potential. The set of offset positions was chosen to obey the Maxwell-Bolzmann distribution at $300 \mathrm{~K}$ with regard to the calculated potential energy. At room temperature the thermal energy is high enough to allow lateral offsets of up to $\sim 2 \AA$. The resulting averaged SSC pattern is shown in Fig. 3(b) where the intensity near the normal emission shows indeed a very poor structure due to the averaging of different possible offsets. This reproduces very well the experimental XPD pattern observed for the multilayer deposited on $\mathrm{Cu}(111)$.
In comparison to $\mathrm{Cu}(111)$, the multilayer on $\mathrm{Au}(111)$ shows an enhanced anisotropy at small polar angles, indicating an increased constrain on the possible offset positions. The remaining structure at $\vartheta \simeq 24^{\circ}$ could be reproduced with SSC by the average pattern of various lateral offsets in the C-C bond direction [Fig. 3(d)]. This preferred offset from the column axis possibly originates from the interaction with neighboring molecules of the same layer. The equilibrium distance between them is $14.1 \AA$ and thus for the column separation on $\mathrm{Au}(111)(15.0 \AA)$ an attractive force exists between these molecules resulting in typical offsets of $\sim 1 \AA$. Larger off-center distances are energetically unfavorable [Fig. 4(b)] which promotes the growth of columnar structures with small lateral offsets.

In conclusion, we have shown that adsorption of $\mathrm{HBC}$ on $\mathrm{Au}(111)$ and $\mathrm{Cu}(111)$ forms ordered molecular monolayer films with a superlattice that is commensurate to the substrate lattice due to a sufficent molecule-substrate interaction. The intermolecular distances on $\mathrm{Au}(111)$ and $\mathrm{Cu}(111)$ are 15.0 and $14.2 \AA$, respectively. Due to the van der Waals interaction subsequently deposited molecules are adsorbed flat near the center of the first-layer molecules. The molecular lattice of the first monolayer is thus transmitted to the following layers of molecules resulting in the growth of columnar stacks with conservation of the molecule orientation and a limited offset from the columnar axis. In that sense, the ordered monolayer system acts as template for the growth of columnar structures with a substrate dependent stack separation. The prospect of this work is the building of scaffoldings made of molecular stacks for the positioning of functional groups.

Financial support by the Swiss National Science Foundation (Grant Nos. NFP 47 and MaNEP) is greatfully acknowledged.
*Electronic address: pascal.ruffieux@unifi.ch

${ }^{1}$ C. Joachim, J.K. Gimzewski, and A. Aviram, Nature (London) 408, 541 (2000).

${ }^{2}$ N. Karl and Ch. Günther, Cryst. Res. Technol. 34, 243 (1999).

${ }^{3}$ T. Yokoyama, S. Yokoyama, T. Kamikado, Y. Okum, and S. Mashiko, Nature (London) 413, 619 (2001).

${ }^{4}$ G.P. Lopinski, D.D.M. Wayner, and R.A. Wolkow, Nature (London) 406, 48 (2000).

${ }^{5}$ P. Herwig, C.W. Kayser, K. Müllen, and H.W. Spiess, Adv. Mater. 8, 510 (1996).

${ }^{6}$ A.V. de Craats, J. Warman, A. Fechtenkötters, J.D. Brandt, and K. Müllen, Adv. Mater. 11, 1469 (1999).

${ }^{7}$ D. Adam, F. Closs, T. Frey, D. Funhoff, D. Haarer, H. Ringsdorf, P. Schuhmacher, and K. Siemensmeyer, Phys. Rev. Lett. 70, 457 (1993).

${ }^{8}$ S. Ito, M. Wehmeier, J.D. Brand, C. Kübel, R. Epsch, J.P. Rabe, and K. Müllen, Chem.-Eur. J. 6, 4327 (2000).

${ }^{9}$ R. Goddard, M.W. Haenel, W.C. Herndon, C. Krüger, and M. Zander, J. Am. Chem. Soc. 117, 30 (1995).
${ }^{10}$ R. Fasel, P. Aebi, R.G. Agostino, D. Naumović, J. Osterwalder, A. Santaniello, and L. Schlapbach, Phys. Rev. Lett. 76, 4733 (1996).

${ }^{11}$ R.M. Küttel, R.G. Agostino, R. Fasel, J. Osterwalder, and L. Schlapbach, Surf. Sci. 312, 131 (1994).

${ }^{12}$ J. Osterwalder, P. Aebi, R. Fasel, D. Naumovic, P. Schwaller, T. Kreutz, L. Schlapbach, T. Abukawa, and S. Kono, Surf: Sci. 331-333, 1002 (1995).

${ }^{13}$ H. Proehl, M. Toerker, F. Sellam, T. Fritz, K. Leo, C. Simpson, and K. Müllen, Phys. Rev. B 63, 205409 (2001).

${ }^{14}$ M. Keil, P. Samorí, D.A. dos Santos, T. Kugler, S. Stafström, J.D, Brand, K. Müllen, J.L. Brédas, J.P. Rabe, and W.R. Salaneck, J, Phys. Chem: B 104, 3967 (2000).

${ }^{15}$ S.J. Stuart, A.B. Tutein, and J.A. Harrison, J. Chem. Phys. 112, 6472 (2000).

${ }^{16}$ J. Song and R.L. Cappelletti, Phys. Rev. B 50, 14678 (1994).

${ }^{17}$ G.H. Hudson and J.C. McCoubrey, Trans. Faraday Soc. 56, 761 (1959). 